PROCEEDINGS OF THE AMERICAN MATHEMATICAL SOCIETY

Volume 124, Number 5, May 1996

\title{
TRACE THEOREMS FOR HOLOMORPHIC SEMIGROUPS AND THE SECOND ORDER CAUCHY PROBLEM
}

\author{
O. EL-MENNAOUI AND V. KEYANTUO
}

(Communicated by Palle E. T. Jorgensen)

\begin{abstract}
We use the theory of boundary values (also called traces) of holomorphic semigroups as developed by Boyadzhiev-deLaubenfels (1993) and El-Mennaoui (1992) to study the second order Cauchy problem for certain generators of holomorphic semigroups. Our results contain in particular the result of Hieber (Math. Ann. 291 (1991), 1-16) for the Laplace operator on $L^{p}\left(\mathbb{R}^{N}\right)$.
\end{abstract}

\section{INTRODUCTION}

In connection with the abstract Cauchy problem $(\mathrm{ACP} 1)$

$$
u^{\prime}(t)=A u(t), \quad t \geq 0 ; \quad u(0)=x
$$

the theory of strongly continuous semigroups is well developed. The Hille-Yosida theorem gives a characterization of those closed linear operators $A$ with domain and range in a Banach space $X$ for which (ACP) is well-posed. Of interest are also Cauchy problems of the second order

$$
u^{\prime \prime}(t)=A u(t), \quad t \geq 0 ; \quad u(0)=x, \quad u^{\prime}(0)=y .
$$

Although (ACP2) may be reduced in a classical way to a first order problem (via the energy space), a direct approach presents some advantages (see Fattorini [10]). Parallel to the theory of strongly continuous semigroups, a theory of strongly continuous cosine functions has been constructed. The corresponding generation theorem was established independently by Da Prato, Sova and Fattorini.

In this paper, we will be concerned with Cauchy problems that are not well-posed in the classical sense. The theory of integrated semigroups was developed recently to treat (ACP1) in some cases where the Hille-Yosida theorem does not apply. The analogous theory for $(\mathrm{ACP} 2)$ is that of integrated cosine functions.

Using the trace theorem for holomorphic semigroups proved in BoyadzhievdeLaubenfels [5] using the language of $C$-regularized semigroups and extended by El-Mennaoui [9] (the latter author uses integrated semigroups), we first prove that the Laplace operator $\Delta$ on $L^{p}\left(\mathbb{R}^{N}\right)$ with maximal distributional domain is the generator of an $\alpha$-times integrated cosine function for any $\alpha>(N-1)\left|\frac{1}{2}-\frac{1}{p}\right|$. This gives an entirely new proof of the result obtained by Hieber [12] by the use of

Received by the editors June 6, 1994 and, in revised form, September 26, 1994.

1991 Mathematics Subject Classification. Primary 47D06, 47F05.

This work was supported by the DAAD and the European Science Plan "Evolutionary Systems". 
Fourier multipliers. Next we prove that if a closed linear operator $A$ generates a holomorphic semigroup of angle $\frac{\pi}{2}$ satisfying a Gaussian estimate, then (ACP2) is governed by an integrated cosine function. We also establish optimality for this result.

Actually, the aforementioned boundary value theorems allow one to pass from the operator $A$ to the operator $i A$; in other words, it allows one to derive solvability of the Schrödinger evolution equation from that of the heat equation. In [17] it has been proved that with the help of the trace theorem, one can solve the Schrödinger equation starting from the wave equation. Hence our result here is a converse. We apply this result to general elliptic operators in $L^{p}(\Omega)$ where $\Omega \subset \mathbb{R}^{N}$ is open, with Dirichlet or Neumann boundary conditions (in the latter case, some regularity on $\Omega$ has to be assumed). We note that as far as the second order Cauchy problem is concerned, our results are new when $\Omega$ has infinite Lebesgue measure. The case where $\Omega$ is of finite measure was considered in [16] using fractional powers of operators and Sobolev imbedding theorems.

The organization of the work is the following. In Section 2, we recall the definition of integrated semigroups and integrated cosine functions as well as the trace theorem which will be of constant use in the sequel. In Section 3, we give the generation result for the Laplace operator on $L^{p}\left(\mathbb{R}^{N}\right)$. In the fourth section, we consider the general case and give a counterexample showing optimality. We also consider as an illustration the Cauchy-Poisson semigroup in one dimension which is related to the Hilbert transform. The fifth and last section is devoted to application to elliptic operators in $L^{p}(\Omega), 1 \leq p<\infty$.

\section{Preliminaries}

In this section, we give the definitions of semigroups and integrated cosine functions and state the trace theorem for holomorphic semigroups. The theory of exponentially bounded $n$-times integrated semigroups was introduced by Arendt (see [1]), see also Neubrander [18]. Later, Hieber [12] extended the definition to $\alpha$-times integrated semigroups, $\alpha \in \mathbb{R}_{+}=[0, \infty)$.

Definition 2.1. Let $\alpha \geq 0$. Then $A$ is the generator of an $\alpha$-times integrated semigroup if $(\omega, \infty) \subset \rho(A)$ for some $\omega \in \mathbb{R}$ and there exists a strongly continuous mapping $S:[0, \infty) \rightarrow \mathcal{L}(X)$ with $\|S(t)\| \leq M e^{\omega t}, t \geq 0$, for some $M \geq 0$ such that

$$
R(\lambda, A)=\lambda^{\alpha} \int_{0}^{\infty} e^{-\lambda t} S(t) d t \quad \text { for } \lambda>\max \{\omega, 0\} .
$$

In this case, $(S(t))$ is called the $\alpha$-times integrated semigroup generated by $A$.

The integral in $(2-1)$ is understood as an improper strong Riemann integral. When $\alpha=0$, then $(S(t))$ is a $C_{0}$-semigroup and $A$ is automatically densely defined. Similarly, we give the definition of $\alpha$-times integrated cosine functions. They were introduced by Arendt-Kellermann [2] for $\alpha \in \mathbb{N}$ and the extension to $\alpha \in[0, \infty)$ is again due to Hieber [12].

Definition 2.2. Let $\alpha \geq 0$. Then $A$ is the generator of an $\alpha$-times integrated cosine function if there exist $M, \omega \in \mathbb{R}$ such that $\left\{\lambda^{2}: \lambda>\omega\right\} \subset \rho(A)$ and there exists a strongly continuous mapping $S:[0, \infty) \rightarrow \mathcal{L}(X)$ with $\|S(t)\| \leq M e^{\omega t}, t \geq 0$, 
such that

$$
R\left(\lambda^{2}, A\right)=\lambda^{\alpha-1} \int_{0}^{\infty} e^{-\lambda t} S(t) d t \quad \text { for } \lambda>\max \{\omega, 0\}
$$

As for the case of Definition 2.1, $(S(t))$ is the $\alpha$-times integrated cosine function generated by $A$. For $\alpha=0,(S(t))$ is the strongly continuous cosine function generated by $A$. The sine function corresponds by definition to the case $\alpha=1$. By the uniqueness theorem for Laplace transforms there exists at most one family $(S(t))$ satisfying either definition. A complete study of cosine functions and their relations to the abstract Cauchy problem may be found in Fattorini [10] or Goldstein [11]. For integrated cosine functions, see [2] or [12].

The classical theorem of M. H. Stone states that in a Hilbert space $H$, a closed linear operator $B$ is the generator of a unitary group if and only if $i B$ is a self-adjoint operator. Let now $A$ be a self-adjoint operator in $H$ satisfying $\sigma(A) \subset(-\infty, \omega)$ for some $\omega \in \mathbb{R}$. Then by functional calculus, $A$ is the generator of a strongly continuous semigroup (of self-adjoint operators) which can be holomorphically continued to the right half-plane $\{z: \operatorname{Re} z>0\}$. Also, $A$ is the generator of a cosine function. From this we have that the following three Cauchy problems are simultaneously well-posed:

$$
\begin{gathered}
u^{\prime}(t)=A u(t), \quad t \geq 0 ; \quad u(0)=x \\
u^{\prime \prime}(t)=A u(t), \quad t \geq 0 ; \quad u(0)=x, \quad u^{\prime}(0)=y \\
u^{\prime}(t)=i A u(t), \quad t \geq 0 ; \quad u(0)=x .
\end{gathered}
$$

If we take $A$ to be the Laplace operator in $L^{2}(\Omega)$ where $\Omega \subset \mathbb{R}^{N}$ is open with, say, Dirichlet boundary conditions, then $(\mathrm{H}),(\mathrm{W})$ and $(\mathrm{S})$ correspond respectively to the heat, wave and Schrödinger equations. If we denote by $T(z), \operatorname{Re} z>0$, the semigroup governing $(\mathrm{H})$ then one has

$$
\|T(z)\| \leq M, \quad \operatorname{Re} z>0
$$

For $X=L^{p}\left(\mathbb{R}^{N}\right), 1 \leq p<\infty, A$ the Laplacian with maximal distributional domain, the situation is quite different. The equation $(\mathrm{H})$ is still governed by a holomorphic semigroup of angle $\frac{\pi}{2}$ but instead of (2-3) we have

$$
\|T(z)\| \leq M\left(\frac{|z|}{\operatorname{Re} z}\right)^{N|1 / 2-1 / p|}, \quad \operatorname{Re} z>0 .
$$

In fact, it was proved by Hörmander [15] that if $p \neq 2$, then $i \Delta$ does not generate a $C_{0}$-semigroup. The results of [15] also imply that $\Delta$ generates a cosine function in $L^{p}\left(\mathbb{R}^{N}\right)$ if and only if $(p=2$ or $N=1$ ) (see e.g. Hieber [12]). This shows the interest in the following theorem from [9].

Theorem 2.3. Let $\beta \geq 0$. Assume that $A$ is densely defined and generates a holomorphic semigroup of angle $\frac{\pi}{2}$. Then the following assertions are equivalent:

(i) For all $\alpha>\beta$, there exist $M, \omega \geq 0$ such that

$$
\|T(z)\| \leq M e^{\omega \operatorname{Re} z}\left(\frac{|z|}{\operatorname{Re} z}\right)^{\alpha}, \quad \operatorname{Re} z>0 .
$$


(ii) For all $\alpha>\beta$ the operator $i A$ generates an $\alpha$-times integrated group $(S(t))$ satisfying

$$
\sup _{t \neq 0} \frac{\|S(t)\|}{|t|^{\alpha}}<\infty .
$$

For the proof, we refer to El-Mennaoui [9, Theorem 5.1, p. 42], and Corollary 5.3, p. 44]. Theorem 2.3 is a refinement of a result of Boyadzhiev and deLaubenfels [5] (see also deLaubenfels [8, Chapter $\mathrm{X}]$ who show that (i) implies that $i A$ generates a $(\lambda-A)^{-\alpha}$-regularized group for all $\alpha>\beta$. For $\alpha$ entire the latter is equivalent to saying that $i A$ generates an $\alpha$-times integrated group.

Other versions of the trace theorem are available in [9] especially when the holomorphic semigroup $(T(z))$ is not assumed a priori to be in the class $C_{0}$. But we shall not need them here; see however Section 5. We note that semigroups satisfying (2-3) in a Banach space were considered by Hille-Phillips (see [13, Theorems 17.9.1 and 17.9.2]). But as we have seen above, this condition is too restrictive for the applications we have in view.

In this paper we consider application of Theorem 2.3 to the second order Cauchy problem.

\section{The Wave Equation on $L^{p}\left(\mathbb{R}^{N}\right)$}

In this section we prove that $\Delta$ generates an $\alpha$-times integrated cosine function on $L^{p}\left(\mathbb{R}^{N}\right)$ when $\alpha>(N-1)\left|\frac{1}{2}-\frac{1}{p}\right|$. This result is due to Hieber [12] who uses multiplier theory for the proof. Our proof is based on Theorem 2.3.

This example is illuminating for the abstract result presented in Section 4 where we also use some formulas developed in this section.

We start with the following simple lemma.

Lemma 3.1. Assume that $B$ is the generator of an $\alpha$-times integrated group $(G(t))$ on $X$. Then $A=B^{2}$ is the generator of an $\alpha$-times integrated cosine function given by

$$
S(t)=\frac{1}{2}(G(t)+G(-t)), \quad t \in \mathbb{R} .
$$

Proof. This follows directly from Definition 2.1 and the resolvent equation. In fact, there exist $M \geq 0, \omega>0$ such that $\|G(t)\| \leq M e^{\omega|t|}(t \in \mathbb{R})$. We have by Definition 2.1

$$
R(\lambda, B)=\lambda^{\alpha} \int_{0}^{\infty} e^{-\lambda t} G(t) d t \quad \text { for } \lambda>\omega
$$

similarly

$$
R(\lambda,-B)=\lambda^{\alpha} \int_{0}^{\infty} e^{-\lambda t} G(-t) d t \quad \text { for } \lambda>\omega .
$$

Hence with $S(\cdot)$ given by (3-1), we have for $\lambda>\omega$

$$
\lambda^{\alpha} \int_{0}^{\infty} e^{-\lambda t} S(t) d t=\frac{1}{2}(R(\lambda, B)+R(\lambda,-B))=\lambda R\left(\lambda, B^{2}\right) .
$$

In view of Definition 2.2, this proves the result. 
Remark. The converse of Lemma 3.1 has been proved by Fattorini [10] for $\alpha=0$ and by Hieber [12] for general $\alpha$. More precisely, let $A$ be the generator of an $\alpha$-times integrated cosine function on $L^{p}(\Omega), \Omega \subset \mathbb{R}^{N}$ open. Then there exist $b \in \mathbb{C}$ and $B$ the generator of an $\alpha$-times integrated group on $L^{p}(\Omega)$ such that $A=B^{2}+b I$.

Now, assume that $A$ generates a $C_{0}$-semigroup $(T(t))$. If $(T(t))$ is uniformly bounded, then one can define the fractional powers $(-A)^{\alpha}$ of $-A$ for $0<\alpha<1$. We restrict ourselves to the case of the square root $(-A)^{1 / 2}$ of $-A$ in the following way. First define the operator $J$ with domain $D(J)=D(A)$ by

$$
J x=\frac{1}{\pi} \int_{0}^{\infty} \lambda^{-1 / 2}(\lambda-A)^{-1}(-A) x d \lambda, \quad x \in D(J) .
$$

Then $J$ is closable and, by definition, $\bar{J}:=(-A)^{1 / 2}$ (see e.g. Yosida [20, p. 260]). One can characterize $(-A)^{1 / 2}$ as follows (see Butzer-Berens [6, p. 156] or Yosida [20, p. 260]):

$$
x \in D\left((-A)^{1 / 2}\right) \Leftrightarrow s-\lim _{\varepsilon \rightarrow 0} \frac{1}{\Gamma(-1 / 2)} \int_{\varepsilon}^{\infty} t^{-3 / 2}(T(t)-I) x d t \text { exists. }
$$

In this case, $(-A)^{1 / 2} x=-s-\lim _{\varepsilon \rightarrow 0} \frac{1}{\Gamma(-1 / 2)} \int_{\varepsilon}^{\infty} t^{-3 / 2}(T(t)-I) x d t$.

The operator $-(-A)^{1 / 2}$ is the generator of a holomorphic semigroup $\left(T_{1 / 2}(t)\right)$ which has an explicit representation (see [20, p. 268]):

$$
T_{1 / 2}(t) x=\frac{t}{2 \sqrt{\pi}} \int_{0}^{\infty} e^{-t^{2} / 4 s} T(s) x \frac{d s}{s^{3 / 2}}, \quad x \in X, t>0 .
$$

After a change of variable we obtain

$$
T_{1 / 2}(t) x=\frac{t^{1 / 2}}{2 \sqrt{\pi}} \int_{0}^{\infty} e^{-t / 4 s} T(t s) x \frac{d s}{s^{3 / 2}}, \quad x \in X, t>0 .
$$

A further change of variable gives

$$
T_{1 / 2}(t) x=\frac{t^{1 / 2}}{2 \sqrt{\pi}} \int_{0}^{\infty} e^{-t s / 4} T(t / s) x \frac{d s}{s^{1 / 2}}, \quad x \in X, t>0 .
$$

Next, we consider the Cauchy-Poisson semigroup in $L^{p}\left(\mathbb{R}^{N}\right)$.

Examples. Consider again the Laplacian $\Delta$ on $L^{p}\left(\mathbb{R}^{N}\right), 1 \leq p<\infty$, with maximal distributional domain. Then the Gaussian semigroup generated by $\Delta$ is given by

$$
T(t) f(x)=(4 \pi t)^{-N / 2} \int_{\mathbb{R}^{N}} e^{-|y|^{2} / 4 t} f(x-y) d y, \quad t>0,
$$

for $f \in X=L^{p}\left(\mathbb{R}^{N}\right)$. 
Substituting into $(3-4)^{\prime \prime}$ we obtain for $t>0$

$$
\begin{aligned}
T_{1 / 2}(t) f(x) & =\frac{t^{1 / 2}}{2 \sqrt{\pi}} \int_{0}^{\infty} e^{-t s / 4} T(t / s) f(x) \frac{d s}{s^{1 / 2}} \\
& =\frac{t^{1 / 2}}{2 \sqrt{\pi}} \int_{0}^{\infty} e^{-t s / 4}\left(4 \pi \frac{t}{s}\right)^{-N / 2} \int_{\mathbb{R}^{N}} e^{-|y|^{2} s / 4 t} f(x-y) d y \frac{d s}{s^{1 / 2}} \\
& =\frac{t^{1 / 2}}{2 \sqrt{\pi}}(4 \pi t)^{-N / 2} \int_{\mathbb{R}^{N}} f(x-y) d y \int_{0}^{\infty} e^{-t s / 4} s^{N / 2} e^{-|y|^{2} s / 4 t} s^{-1 / 2} d s \\
& =\frac{t^{1 / 2-N / 2}}{2 \sqrt{\pi}}(4 \pi)^{-N / 2} \int_{\mathbb{R}^{N}} f(x-y) d y \int_{0}^{\infty} s^{(N-1) / 2} e^{-\left(t+|y|^{2} / t\right) \frac{s}{4}} d s \\
& =\pi^{-(N+1) / 2} \Gamma\left(\frac{N+1}{2}\right) t \int_{\mathbb{R}^{N}} \frac{f(x-y)}{\left(t^{2}+|y|^{2}\right)^{(N+1) / 2}} d y .
\end{aligned}
$$

Actually, this formula is well known (see e.g. Stein [19, p. 60] where it is studied in connection with $N$-dimensional singular integrals). The case $N=1$ is studied in Butzer-Berens [6, Chapter 4]. We shall prove the following.

Proposition 3.2. The Laplacian $\Delta$ on $L^{p}\left(\mathbb{R}^{N}\right), 1 \leq p<\infty$, with maximal distributional domain is the generator of an $\alpha$-times integrated cosine function for any $\alpha>(N-1)\left|\frac{1}{2}-\frac{1}{p}\right|$.

Before proving this proposition, we remark that for $N=1, \Delta$ generates the cosine function $C(t)$ given by d'Alembert's formula

$$
C(t) f(x)=\frac{1}{2}(f(x+t)+f(x-t)), \quad f \in L^{p}(\mathbb{R}), x, t \in \mathbb{R} .
$$

In the proof of Proposition 3.2, we may therefore omit this case. However, we need the corresponding estimate for the remark following Proposition 3.4.

Proof of Proposition 3.2. It suffices to prove that the semigroup $T_{1 / 2}(z)$ generated by $-(-\Delta)^{1 / 2}$ satisfies the estimate in Theorem 2.3. We apply the Riesz-Thorin interpolation theorem.

Let us denote momentarily the corresponding semigroup in $L^{p}\left(\mathbb{R}^{N}\right)$ by $T_{1 / 2}^{p}(z)$. Then these semigroups are consistent. This means that $T_{1 / 2}^{p}(t) f=$ $T_{1 / 2}^{q}(t) f$ for $f \in L^{p} \cap L^{q}$. Moreover $\left(T_{1 / 2}^{p}(z)\right)^{*}=T_{1 / 2}^{q}(\bar{z})$ for $1 \leq p \leq 2, \frac{1}{p}+\frac{1}{q}=1$ and $\operatorname{Re} z>0$, where $U^{*}$ denotes the adjoint of $U$ for $U \in \mathcal{L}(X)$. So we may consider only $1 \leq p \leq 2$.

In $L^{2}\left(\mathbb{R}^{N}\right)$, by self-adjointness, the semigroup satisfies $\sup _{\operatorname{Re} z>0}\left\|T_{1 / 2}(z)\right\|<\infty$.

We establish the estimate in $L^{1}\left(\mathbb{R}^{N}\right)$. The operator $T(z)$ is given by the above formula with $t$ being replaced by $z$. The operator $T_{1 / 2}(z)$ is of convolution type in $L^{1}\left(\mathbb{R}^{N}\right)$ and hence its norm is given by

$$
\left\|T_{1 / 2}(z)\right\|_{1}=\mathrm{const}|z| \int_{\mathbb{R}^{N}} \frac{d y}{\left.\left.\left|z^{2}+\right| y\right|^{2}\right|^{(N+1) / 2}}, \quad \operatorname{Re} z>0 .
$$

Using spherical coordinates in $\mathbb{R}^{N}$, we obtain

$$
\left\|T_{1 / 2}(z)\right\|_{1}=\mathrm{const} \int_{0}^{\infty} \frac{r^{N-1} d r}{\left.\left.\left|z^{2}+\right| r\right|^{2}\right|^{(N+1) / 2}}, \quad \operatorname{Re} z>0 .
$$


Now the change of variable $r \rightarrow \frac{r}{|z|}$ yields

$$
\begin{aligned}
\left\|T_{1 / 2}(z)\right\|_{1} & \leq \text { const } \int_{0}^{\infty} \frac{r^{N-1} d r}{\left.\left.\left|\left(\frac{z}{|z|}\right)^{2}+\right| r\right|^{2}\right|^{(N+1) / 2}} \\
& =\operatorname{const} \int_{0}^{\infty} \frac{r^{N-1} d r}{\left|e^{2 i \theta}+r^{2}\right|^{(N+1) / 2}} \\
& =\operatorname{const} \int_{0}^{\infty} \frac{r^{N-1} d r}{\left|r^{4}+2 r^{2} \cos 2 \theta+1\right|^{(N+1) / 4}} \\
& =\operatorname{const}\left\{\int_{0}^{1-\cos \theta}+\int_{1-\cos \theta}^{1+\cos \theta}+\int_{1+\cos \theta}^{2}+\int_{2}^{\infty}\right\} \\
& =\operatorname{const}\left(I_{1}+I_{2}+I_{3}+I_{4}\right)
\end{aligned}
$$

where $\theta=\operatorname{Arg} z$. For the estimate, it is sufficient to consider $\pi / 4 \leq \theta<\pi / 2$. We will consider each term separately.

(1)

$$
I_{4} \leq \int_{2}^{\infty} \frac{r^{N-1} d r}{\left|r^{4}-2 r^{2}+1\right|^{(N+1) / 4}}=M_{1}<\infty
$$

$$
\begin{aligned}
I_{3} & \leq 2^{N-1} \int_{1+\cos \theta}^{2} \frac{d r}{\left(r^{4}-2 r^{2}+1\right)^{(N+1) / 4}} \\
& \leq \mathrm{const} \int_{1+\cos \theta}^{2} \frac{d r}{(r-1)^{(N+1) / 2}} .
\end{aligned}
$$

Therefore we have $I_{3} \leq \operatorname{const}\left(\frac{1}{\cos \theta}\right)^{(N-1) / 2}$ if $N>1$ and $I_{3} \leq \operatorname{const} \ln \left(1+\frac{1}{\cos \theta}\right)$ if $N=1$.

(3) For $0<r \leq 2$, one proves by an easy computation that $r^{4}+2 r^{2} \cos 2 \theta+1=$ $\left|e^{2 i \theta}+r^{2}\right|^{2} \geq \sin 2 \theta\left(r \geq 0, \theta \in\left[\frac{\pi}{4}, \frac{\pi}{2}\right]\right)$. Hence

$$
\begin{aligned}
I_{2} & \leq \int_{1-\cos \theta}^{1+\cos \theta} \frac{r^{N-1} d r}{(\sin 2 \theta)^{(N+1) / 2}} \\
& \leq \operatorname{const} \cos \theta(\sin 2 \theta)^{-(N+1) / 2} \leq \operatorname{const}(\cos \theta)^{-(N-1) / 2} .
\end{aligned}
$$

$(4)$

$$
I_{1} \leq \int_{0}^{1-\cos \theta} \frac{r^{N-1} d r}{\left|r^{4}-2 r^{2}+1\right|^{(N+1) / 4}} \leq \mathrm{const} \int_{0}^{1-\cos \theta} \frac{d r}{(-r+1)^{(N+1) / 2}}
$$

and as above, we obtain $I_{1} \leq \operatorname{const}\left(\frac{1}{\cos \theta}\right)^{(N-1) / 2}$ if $N>1$ and $I_{1} \leq \operatorname{const} \ln \left(\frac{1}{\cos \theta}\right)$ for $N=1$.

By the Riesz-Thorin interpolation theorem, Theorem 2.3 and Lemma 3.1, the proposition is proved.

For the approach to this result using Fourier multipliers, see Hieber [12].

Next we prove that in $L^{1}(\mathbb{R}), i(-\Delta)^{1 / 2}$ is not a $C_{0}$-semigroup generator. For the proof, we need the following lemma. 
Lemma 3.3. Assume that the operator $A$ generates a holomorphic semigroup $(T(z))$ of angle $\frac{\pi}{2}$ and that $i A$ generates a $C_{0}$-semigroup $(S(t))$. Let $\tau>0$. Then for all $s \in[0, \tau]$,

$$
\lim _{t \rightarrow 0} T(t+i s) x=S(s) x \quad \text { for all } x \in X .
$$

In particular, for all $R>0$,

$$
\sup _{\operatorname{Re} z>0,|z| \leq R}\|T(z)\|<\infty .
$$

Proof. We first note that for the operator $A$, Ljubich's uniqueness condition

$$
\limsup _{\lambda \rightarrow+\infty} \frac{1}{\lambda} \ln \|R(\lambda, A)\|=0
$$

holds. It follows that the Cauchy problem

$$
u^{\prime}(t)=A u(t), \quad 0 \leq t \leq \tau ; \quad u(0)=0
$$

has at most one classical solution. The same is valid if we replace $A$ by $i A$. Let $x \in D(A)$. Denote by $U(i t) x$ for $t \in[0, \tau]$ the solution of

$$
u^{\prime}(t)=i A u(t), \quad 0 \leq t \leq \tau ; \quad u(0)=x .
$$

Let $W(t, s) x:=T(t+i s) x-T(t) U(i s) x, s \in[0, \tau]$. Then $W(t, 0) x=0$. We have

$$
\begin{aligned}
\frac{d}{d s} W(t, s) x & =i A T(t+i s) x-i A T(t) U(i s) x \\
& =i A(T(t+i s) x-T(t) U(i s) x) \\
& =i A W(t, s) x .
\end{aligned}
$$

From the uniqueness theorem, it follows that

$$
T(t+i s) x=T(t) U(i s) x
$$

for $x \in D(A), t>0,0 \leq s \leq \tau$. But $U(i s) x=S(s) x$ for $x \in D(A)$. It follows that (3-6) holds for $x \in D(A)$ which is dense in $X$. The convergence for arbitrary $x$ follows by the uniform boundedness theorem.

We remark that this lemma can be generalized to the case where the boundary value is an $\alpha$-times integrated semigroup. By the uniform boundedness theorem, one also sees that the convergence in (3-6) is uniform in $t$ on compact subsets of $[0, \infty)$.

Proposition 3.4. The operator $i(-\Delta)^{1 / 2}$ on $L^{1}\left(\mathbb{R}^{1}\right)$ does not generate a $C_{0^{-}}$ semigroup.

Proof. We have by the above formula

$$
\left\|T_{1 / 2}(z)\right\|_{1}=\mathrm{const} \int_{0}^{\infty} \frac{d r}{\left|r^{4}+2 r^{2} \cos 2 \theta+1\right|^{1 / 2}}
$$

where const is a positive constant.

Assume that there exists a strong $\operatorname{limit}_{\mathrm{s}} \lim _{\theta \rightarrow \pi / 2} T_{1 / 2}\left(e^{i \theta}\right)$. Then by the uniform boundedness theorem $\liminf _{\theta \rightarrow \pi / 2}\left\|T_{1 / 2}\left(e^{i \theta}\right)\right\|_{1}<\infty$. 
But by Fatou's lemma

$$
\begin{aligned}
\liminf _{\theta \rightarrow \pi / 2}\left\|T_{1 / 2}\left(e^{i \theta}\right)\right\|_{1} & =\text { const } \liminf _{\theta \rightarrow \pi / 2} \int_{0}^{\infty} \frac{d r}{\left|r^{4}+2 r^{2} \cos 2 \theta+1\right|^{1 / 2}} \\
& \geq \text { const } \int_{0}^{\infty} \frac{d r}{\left|r^{4}-2 r^{2}+1\right|^{1 / 2}}=\infty,
\end{aligned}
$$

which contradicts Lemma 3.3.

Remark. In the case $1<p<\infty$ the situation is different. Fattorini's result says that $i(-\Delta)^{1 / 2}$ on $L^{p}(\mathbb{R})$ does generate a $C_{0}$-semigroup. We give here a direct proof of this fact. Let $A=\frac{\partial^{2}}{\partial x^{2}}$ with domain $W^{2, p}(\mathbb{R})$ in $X=L^{p}(\mathbb{R}), 1 \leq p<\infty$. $A$ is the generator of the cosine function given by the d'Alembert formula

$$
C(t) f(x)=\frac{1}{2}(f(x+t)+f(x-t)), \quad f \in X, x, t \in \mathbb{R} .
$$

The associated sine function (the once integrated cosine function) is

$$
S(t) f(x)=\frac{1}{2} \int_{0}^{t} C(s) f(x) d s=\frac{1}{2} \int_{x-t}^{x+t} f(s) d s, \quad f \in X, x, t \in \mathbb{R} .
$$

Let $(H f)(x)=\lim _{\varepsilon \rightarrow 0} \int_{|s| \geq \varepsilon} \frac{1}{s} f(x-s) d s=$ p.v. $\int_{\mathbb{R}} \frac{f(s)}{x-s} d s$ be the Hilbert transform where p.v. denotes the principal value in the sense of Cauchy. Then by the theorem of M. Riesz (see [6, Theorem 4.2.5, p. 244] or [19, p. 38]), $H \in \mathcal{L}\left(L^{p}(\mathbb{R})\right.$ ) for $1<p<\infty$. The generator of the Poisson semigroup is $-(-A)^{1 / 2}$ where $A$ is the Laplace operator $\Delta$ with maximal distributional domain on $L^{p}(\mathbb{R})$ and we have

$$
-(-A)^{1 / 2} f(x)=H\left(\frac{\partial f}{\partial x}(x)\right)=\frac{\partial}{\partial x}(H f(x)) .
$$

Moreover, $D\left((-A)^{1 / 2}\right)=W^{1, p}(\mathbb{R}), 1<p<\infty($ see $[6$, Chapter 4, p. 248]). An easy computation yields

$$
(-A)^{1 / 2} S(t) f(x)=\frac{1}{2} H(f(x+t)-f(x-t)) .
$$

From the boundedness of the Hilbert transform, it follows that $(-A)^{1 / 2} S(t)$ is a strongly continuous $\mathcal{L}\left(L^{p}(\mathbb{R})\right.$ )-valued mapping. Using Laplace transform, one easily verifies that

$$
U(t)=C(t)+i(-A)^{1 / 2} S(t)
$$

is a strongly continuous group with generator $i(-A)^{1 / 2}$.

We have thus proved that $\left\{T_{1 / 2}(z), \operatorname{Re} z>0\right\}$ admits a boundary value which is a bounded strongly continuous group. From [13, Theorem 17.9.1 and 17.9.2] it follows that $\left\{T_{1 / 2}(z), \operatorname{Re} z>0\right\}$ satisfies $(2-3)$.

\section{The second order Cauchy problem: The general Case}

Using the representation (3-4) of Section 3, we shall prove the following.

Theorem 4.1. Let $\alpha>0$. Assume that $A$ generates a holomorphic semigroup $(T(z))$ of angle $\frac{\pi}{2}$ satisfying

$$
\|T(z)\| \leq M\left(\frac{|z|}{\operatorname{Re} z}\right)^{\alpha}, \quad \operatorname{Re} z>0 .
$$


Then $-(-A)^{1 / 2}$ is the generator of holomorphic semigroup $\left(T_{1 / 2}(z)\right)$ of angle $\frac{\pi}{2}$ satisfying

$$
\left\|T_{1 / 2}(z)\right\|<M^{\prime}\left(\frac{|z|}{\operatorname{Re} z}\right)^{\alpha+1 / 2}, \quad \operatorname{Re} z>0 .
$$

Consequently, A generates a $\beta$-times integrated cosine function for any $\beta>\alpha+1 / 2$.

Proof. It follows from (i) that $\sup _{t>0}\|T(t)\|<\infty$ and so one can define $(-A)^{1 / 2}$ and the corresponding semigroup is given by $(3-4)$ :

$$
T_{1 / 2}(t) x=\frac{t^{1 / 2}}{2 \sqrt{\pi}} \int_{0}^{\infty} e^{-t / 4 s} T(t s) x \frac{d s}{s^{3 / 2}}, \quad x \in X, t>0 .
$$

From this it is plain that $\left(T_{1 / 2}(t)\right)$ can be continued holomorphically to the right half-plane $\{z: \operatorname{Re} z>0\}$.

$$
T_{1 / 2}(z) x=\frac{z^{1 / 2}}{2 \sqrt{\pi}} \int_{0}^{\infty} e^{-z / 4 s} T(z s) x \frac{d s}{s^{3 / 2}}, \quad x \in X, \operatorname{Re} z>0,
$$

where we choose $z^{1 / 2}$ to be positive when $z$ is positive. We now estimate $\left\|T_{1 / 2}(z)\right\|$ :

$$
\begin{aligned}
\left\|T_{1 / 2}(z)\right\| & =\frac{|z|^{1 / 2}}{2 \sqrt{\pi}}\left\|\int_{0}^{\infty} e^{-z / 4 s} T(z s) \frac{d s}{s^{3 / 2}}\right\| \\
& \leq \frac{|z|^{1 / 2}}{2 \sqrt{\pi}} \int_{0}^{\infty} e^{-\operatorname{Re} z / 4 s}\|T(z s)\| \frac{d s}{s^{3 / 2}} \\
& \leq \frac{|z|^{1 / 2}}{2 \sqrt{\pi}} M\left(\frac{|z|}{\operatorname{Re} z}\right)^{\alpha} \int_{0}^{\infty} e^{-\operatorname{Re} z / 4 s} \frac{d s}{s^{3 / 2}} \\
& \leq \frac{|z|^{1 / 2}}{2 \sqrt{\pi}} M\left(\frac{|z|}{\operatorname{Re} z}\right)^{\alpha} \frac{1}{(\operatorname{Re} z)^{1 / 2}} \int_{0}^{\infty} e^{-1 / 4 s} \frac{d s}{s^{3 / 2}} \\
& =\operatorname{const}\left(\frac{|z|}{\operatorname{Re} z}\right)^{\alpha+1 / 2} .
\end{aligned}
$$

By application of Theorem 2.3, we obtain that $i(-A)^{1 / 2}$ is the generator of a $\beta$-times integrated group for any $\beta>\alpha+1 / 2$. It now follows from Lemma 3.1 that $A$ generates a $\beta$-times integrated cosine function for $\beta>\alpha+1 / 2$.

Remark 1. An $\alpha$-times integrated group $(S(t))$ satisfying the estimate

$$
\sup _{t \neq 0} \frac{\|S(t)\|}{|t|^{\alpha}}<\infty
$$

is called tempered. Such integrated groups have been studied in [9]. See also Balabane, Emamirad and Jazar [4] where tempered integrated semigroups are studied using the theory of spectral distributions of Colojoara and Foias.

Similarly, if $(S(t))$ is an $\alpha$-times integrated cosine function satisfying the above estimate (4-2), then we say that it is tempered. It follows from the above proof that the integrated cosine functions we obtain in Proposition 3.2 and Theorem 4.1 are tempered.

Remark 2. Let $(T(t))$ be a bounded semigroup. By Yosida [20, Chapter IX, pp. 263-264], if $A$ is the generator of $(T(t))$, then the fractional powers $-(-A)^{\alpha}, 0<$ $\alpha<1$, generate holomorphic semigroups. The explicit representation $(2-5)$ gives that for $\alpha=1 / 2$, the angle of the corresponding semigroup is at least $\frac{\pi}{4}$. In our case, 
$(T(t))$ is holomorphic of angle $\frac{\pi}{2}$ and $(2-5)$ gives the same angle for the semigroup generated by $-(-A)^{1 / 2}$.

Example 1. Denote by $\mathbb{C}_{+}$the right half-plane $\{z: \operatorname{Re} z>0\}$ and $H\left(\mathbb{C}_{+}\right)$the space of functions holomorphic in $\mathbb{C}_{+}$. Consider the Banach space

$$
X:=\left\{f \in C \overline{\left(\mathbb{C}_{+}^{b}\right)} \cap H\left(\mathbb{C}_{+}\right) ; \lim _{|z| \rightarrow \infty}|f(z)|=0\right\}
$$

with supremum norm.

Let $(T(z))$ be defined by

$$
T(z) f(\zeta)=f(z+\zeta), \quad \operatorname{Re} z \geq 0, \operatorname{Re} \zeta \geq 0 .
$$

Then $(T(z))$ is a strongly continuous holomorphic semigroup of angle $\frac{\pi}{2}$. Moreover, we have $\|T(z)\| \leq 1, \operatorname{Re} z>0$. Let $0<\alpha<1 / 2$ and

$$
f_{\alpha}(\zeta)=\frac{\zeta^{\alpha}}{1+\zeta} e^{-1 / \zeta}
$$

Then from (3-4)' above, we have

$$
T_{1 / 2}(z) f_{\alpha}(\zeta)=\text { const } \frac{z^{1 / 2}}{2 \sqrt{\pi}} \int_{0}^{\infty} e^{-z / 4 s} f_{\alpha}((\zeta+z) s) \frac{d s}{s^{3 / 2}}
$$

and after another change of variable

$$
T_{1 / 2}(t) x=\frac{t^{1 / 2}}{2 \sqrt{\pi}} \int_{0}^{\infty} e^{-t s / 4} T(t / s) x \frac{d s}{s^{1 / 2}}, \quad x \in X, \operatorname{Re} z>0 .
$$

Here again, const is a positive constant. Throughout the proof, const may vary without depending on $\alpha$ and $z$.

It follows that

$$
T_{1 / 2}(z) f_{\alpha}(\zeta)=\mathrm{const} \frac{z^{1 / 2}}{2 \sqrt{\pi}} \int_{0}^{\infty} e^{-z s / 4} \frac{e^{-1(\zeta+z / s)}}{1+\zeta+\frac{z}{s}}\left(\zeta+\frac{z}{s}\right)^{\alpha} \frac{d s}{s^{1 / 2}}, \quad \operatorname{Re} z>0 .
$$

Therefore if $|z|=1$, then

$$
\begin{aligned}
\left\|T_{1 / 2}(z) f_{\alpha}\right\| & \geq\left|T_{1 / 2}(z) f_{\alpha}(0)\right| \\
& =\operatorname{const}\left|\frac{z^{1 / 2}}{2 \sqrt{\pi}} \int_{0}^{\infty} e^{-z s / 4} \frac{e^{-s / z}}{1+\frac{z}{s}}\left(\frac{z}{s}\right)^{\alpha} \frac{d s}{s^{1 / 2}}\right| \\
& =\frac{|z|^{1 / 2}|z|^{\alpha}}{\left|z+\frac{1}{z}\right|^{1 / 2-\alpha}} \int_{0}^{\infty} e^{-s} s^{-1 / 2-\alpha}\left|1+\frac{z}{s}\left(z+\frac{1}{z}\right)\right|^{-1} d s \\
& \geq \frac{|z|^{1 / 2+\alpha}}{2(\operatorname{Re} z)^{1 / 2-\alpha}} \int_{0}^{\infty} e^{-s} s^{-1 / 2-\alpha} d s \\
& =\operatorname{const} \frac{1}{(\operatorname{Re} z)^{1 / 2-\alpha}}
\end{aligned}
$$

where we have assumed $|z|=1$ so that $z+\frac{1}{z}$ becomes real. It is easily verified that $\left\|f_{\alpha}\right\| \leq 1$. Therefore for $|z|=1$, we have

$$
\left\|T_{1 / 2}(z)\right\| \geq \frac{\left\|T_{1 / 2}(z) f_{\alpha}\right\|}{\left\|f_{\alpha}\right\|} \geq \operatorname{const}(\operatorname{Re} z)^{\alpha-1 / 2} .
$$

It follows from Theorem 2.3 ((i) $\Rightarrow$ (ii)) that our result is optimal, since in the example, $\alpha$ can be chosen arbitrary in $(0,1 / 2)$. 


\section{Application to the Wave equation in $L^{p}(\Omega)$}

In this section, we apply Theorem 4.1 to elliptic operators with variable coefficients.

Let $\Omega \subset \mathbb{R}^{N}$ be open and $\left(a_{i j}(x)\right), 1 \leq i, j \leq N$, be real valued functions belonging to $L_{\text {loc }}^{\infty}(\Omega)$ and such that $a_{i j}=a_{j i}$. As a general reference, see [7]. We assume that there are two positive constants $\lambda$ and $\mu$ such that the following ellipticity condition is satisfied:

$$
0<\lambda|\psi|^{2} \leq \sum_{1 \leq i, j \leq N} a_{i j}(x) \psi_{i} \overline{\psi_{j}} \leq \mu|\psi|^{2}, \quad \psi \in \mathbb{C}^{N} \backslash\{0\}, \text { a.e. in } \Omega .
$$

Consider the two nonnegative symmetric quadratic forms $a$ and $b$ below:

$$
\begin{gathered}
a: H^{1}(\Omega) \times H^{1}(\Omega) \rightarrow \mathbb{C}, \\
a(u, v)=\sum_{1 \leq i, j \leq N}\left(a_{i j}(x) \frac{\partial u}{\partial x_{i}}, \frac{\partial v}{\partial x_{j}}\right)_{L^{2}(\Omega)}
\end{gathered}
$$

and

$$
\begin{gathered}
b: H_{0}^{1}(\Omega) \times H_{0}^{1}(\Omega) \rightarrow \mathbb{C}, \\
b(u, v)=\sum_{1 \leq i, j \leq N}\left(a_{i j}(x) \frac{\partial u}{\partial x_{i}}, \frac{\partial v}{\partial x_{j}}\right)_{L^{2}(\Omega)} .
\end{gathered}
$$

The forms $a$ and $b$ correspond respectively to Dirichlet and Neumann boundary conditions. In symbols, we use $D$ and $N e$. We denote by $A_{2}^{D}$ and $A_{2}^{N e}$ the associated self-adjoint operators in $L^{2}(\Omega)$. In the following, $\Omega$ may be an arbitrary open subset of $\mathbb{R}^{N}$ when we consider Dirichlet boundary conditions; but we assume that $\Omega$ has the extension property (e.g. $\Omega$ may be of class $C^{1}$ ) in the case where we deal with Neumann boundary conditions.

The semigroup $\left(T_{2}(t)\right)$ generated by $A_{2}^{D}$ or $A_{2}^{N e}$ possesses a kernel $K$ satisfying

$$
0 \leq K(t, x, y) \leq c(4 \pi t)^{-N / 2} \exp \left(-d(x-y)^{2} / 4 t\right), \quad 0<t \leq 1, x, y \in \Omega,
$$

where $c$ and $d$ are positive constants (see [7]). Actually, the semigroups can be extended analytically to $\{z: \operatorname{Re} z>0\}$ and in [7, p. 103] it is proved that the kernel satisfies the complex estimate

$$
|K(z, x, y)| \leq c(4 \pi \operatorname{Re} z)^{-N / 2} \exp \left(-\operatorname{Re}\left[d(x-y)^{2} / 4 z\right]\right),
$$

$\operatorname{Re} z>0, x, y \in \Omega$.

Using (2-4) and the Riesz interpolation theorem, the following proposition is proved in [9, Proposition 6.1, p. 48].

Proposition 5.1. For $1 \leq p<\infty$, there exists a $C_{0}$-holomorphic semigroup of angle $\pi / 2\left(T_{p}(t)\right)$ in $L^{p}(\Omega)$ satisfying $T_{p}(t) f=T_{2}(t) f$ for $f \in L^{p}(\Omega) \cap L^{2}(\Omega)$. Moreover

$$
\left\|T_{p}(z)\right\| \leq M e^{\omega \operatorname{Re} z}\left(\frac{|z|}{\operatorname{Re} z}\right)^{2 N|1 / 2-1 / p|}, \quad \operatorname{Re} z>0 .
$$

In applications, using this proposition, we may take $\omega=0$ because all the families we consider are stable under perturbations by multiples of the identity operator. Combining Theorem 2.3, Theorem 4.1 and Proposition 5.1 we obtain the following. 
Theorem 5.2. Let $1<p<\infty$ and $-A_{p}$ be the infinitesimal generator of $\left(T_{p}(t)\right)$. Then $-A_{p}$ is the generator of an $\alpha$-times integrated cosine function in $L^{p}(\Omega)$ for any $\alpha$ satisfying $\alpha>2 N\left|\frac{1}{2}-\frac{1}{p}\right|+\frac{1}{2}$.

Remark. In the case where $\Omega$ has finite Lebesgue measure, the above constants can be considerably improved. The semigroup $T_{p}(z)$ of Proposition 5.1 now satisfies the estimate

$$
\left\|T_{p}(z)\right\| \leq M e^{\omega \operatorname{Re} z}\left(\frac{1}{\operatorname{Re} z}\right)^{(N / 2)|1 / 2-1 / p|}, \quad \operatorname{Re} z>0 .
$$

This is established in [9, Proposition 6.3, p. 49] using the fact that in this case, $L^{\infty}(\Omega) \subset L^{p}(\Omega)$.

In this case we can use the following version of the trace theorem.

Theorem 5.3 (El-Mennaoui [9, Theorem 4.1, p. 38]). Let $\beta \geq 0$. Assume that $A$ is the generator of a holomorphic semigroup $(T(z))$ of angle $\frac{\pi}{2}$. Then the following assertions are equivalent.

(a) For all $\alpha>\beta$, there exist $M, \omega \geq 0$ such that

$$
\|T(z)\| \leq M e^{\omega|z|}(\operatorname{Re} z)^{-\alpha}, \quad \operatorname{Re} z>0 .
$$

(b) For all $\alpha>\beta$, iA generates an $\alpha$-times integrated group $(S(t))$ on $X$.

Combining these two facts we obtain the following:

Theorem 5.4. Let $1<p<\infty$ and let $-A_{p}$ be the infinitesimal generator of $\left(T_{p}(t)\right)$ as in Proposition 5.1. Assume moreover that $\Omega$ has finite Lebesgue measure. Then $-A_{p}$ is the generator of an $\alpha$-times integrated cosine function in $L^{p}(\Omega)$ for any $\alpha$ satisfying $\alpha>\frac{N}{2}\left|\frac{1}{2}-\frac{1}{p}\right|+\frac{1}{2}$.

Proof. Consider the extension of (3-4)" (giving the semigroup $\left(T_{p}^{1 / 2}(t)\right)$ generated by the square root of the generator of $\left.\left(T_{p}(t)\right)\right)$ to $\{z: \operatorname{Re} z>0\}$. As already noticed above, we may take $\omega=0$ in (5-5). Using (5-5) and this representation, we obtain an estimate for $\left\{\left(T_{p}^{1 / 2}(z)\right), \operatorname{Re} z>0\right\}$. Application of Theorem 5.3 yields the result. We omit the details of the computations.

We note that no optimality result is known in general for the above constants.

\section{ACKNOWLEDGMENTS}

We thank Professor W. Arendt of Université de Franche-Comte for many useful suggestions.

This work was done while the second named author was on a research visit at the Mathematisches Institut der Universität Tübingen. He is grateful to the members of the Functional Analysis group for their kind hospitality.

\section{REFERENCES}

1. W. Arendt, Vector-valued Laplace transforms and Cauchy problems, Israel. J. Math. 59 (1987), 327-352. MR 89a:47064

2. W. Arendt and H. Kellermann, Integrated solutions of Volterra integrodifferential equations and Cauchy problems, Integrodifferential Equations (Proc. Conf. Trento, 1987) (G. Da Prato and M. Iannelli, eds.), Pitman Res. Notes Math. Ser., vol. 190, Longman Sci. Tech., Harlow, 1987, pp. 21-51. MR 90d:00047 
3. W. Arendt, Sobolev imbeddings and integrated semigroups, 2nd International Conference on Trends in Semigroup Theory and Evolution Equations (Ph. Clément, E. Mitidieri, and B. de Pagter, eds.), Lecture Notes in Pure and Appl. Math., vol. 135, Marcel Dekker, New York, 1991. MR 92m: 47001

4. M. Balabane, H. Emamirad, and M. Jazar Spectral distributions and generalization of Stone's theorem, Acta Appl. Math. 31 (1993), 275-295. MR 94f:47038

5. K. Boyadzhiev and R. deLaubenfels Boundary values of holomorphic semigroups, Proc. Amer. Math. Soc. 118 (1993), 113-119. MR 93f:47043

6. P. L. Butzer and H. Berens, Semigroups of operators and approximation, Springer Verlag, Berlin and New York, 1967. MR 37:5588

7. E. B. Davies, Heat kernels and spectral theory, Cambridge Univ. Press, London and New York, 1989. MR 90e:35123

8. R. deLaubenfels, Existence families, functional calculi and evolution equations, Lecture Notes in Math., vol. 1570, Springer Verlag, Berlin and New York, 1994. CMP 94:17

9. O. El-Mennaoui, Traces de semi-groupes holomorphes singuliers à l'origine et comportement asymptotique, Thèse, Besançon, 1992.

10. H. O. Fattorini, Second order linear differential equations in Banach spaces North-Holland, Amsterdam, New York, and London, 1985. MR 87b:34001

11. J. A. Goldstein, Semigroups of linear operators and applications, Oxford Math. Monographs, Oxford Univ. Press, New York, 1985. MR 87c:47056

12. M. Hieber, Integrated semigroups and differential operators on $L^{p}\left(\mathbb{R}^{N}\right)$-spaces, Math. Ann. 291 (1991), 1-16. MR 92g:47052

13. E. Hille and R. S. Phillips, Functional analysis and semigroups, Amer. Math. Soc. Colloq. Publ., vol. 31, Amer. Math. Soc., Providence, RI, 1957. MR 19:664d

14. R. W. Hoppe, Interpolation of cosine operator functions, Ann. Mat. Pura Appl. 136 (1984), 183-212. MR 86b:47074

15. L. Hörmander, Estimates for translation invariant operators in $L^{p}$ spaces, Acta. Math. 104 (1960), pages 93-140. MR 22:12389

16. V. Keyantuo A note on interpolation of semigroups, Proc. Amer. Math. Soc. 123 (1995), 2123-2132. MR 95i:47073

17.

18. F. Neubrander Integrated semigroups and their applications to the abstract Cauchy problem, Pacific J. Math. 135 (1988), 233-251. MR 90b:47073

19. E. M. Stein, Singular integrals and differentiability properties of functions, Princeton Univ. Press, Princeton, NJ, 1971.

20. K. Yosida, Functional analysis, Springer Verlag, Berlin and New York, 1980. MR 82i:46002

Mathematisches Institut der Universität TüBingen, Auf der Morgenstelle 10, 7400 Tübingen, Germany

Équipe de Mathématiques, Université de Franche-Comté, Route de Gray, 25030 Besançon, France

Current address: Department of Mathematics, University of Puerto Rico, Box 23355 Rio Piedras, Puerto Rico 00931 